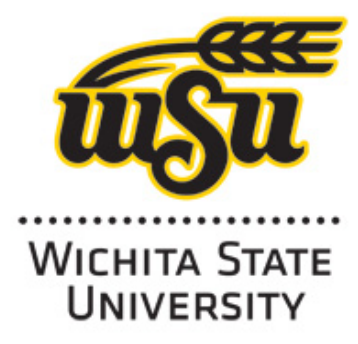

Wichita State University Libraries SOAR: Shocker Open Access Repository

\title{
Interlimb Differences in Lower Extremity Bone Mineral Density Following Anterior Cruciate Ligament Reconstruction
}

\author{
Michael P. Reiman \\ Michael E. Rogers \\ Robert C. Manske
}

Authors' affiliation: Wichita State University

\section{Recommended citation}

Reiman, Michael P., Rogers, Michael E. and Robert C. Manske. 2006. Interlimb differences in lower extremity bone mineral density following anterior cruciate ligament reconstruction. Journal of Orthopaedic \& Sports Physical Therapy, Vol. 36, No.11, pp.837-44. DOI: 10.2519/jospt.2006.2278

This paper is posted in Shocker Open Access Repository

http://soar.wichita.edu/dspace/handle/10057/3687 


\title{
Interlimb Differences in Lower Extremity Bone Mineral Density Following Anterior Cruciate Ligament Reconstruction
}

\author{
Michael P. Reiman, PT, MEd, ATC, CSCS ${ }^{1,2}$ \\ Michael E. Rogers, PhD, CSCS, FACSM ${ }^{3}$ \\ Robert C. Manske, PT, DPT, MEd, SCS, ATC, CSCS ${ }^{1,2}$
}

Study Design: Prospective descriptive study.

Objective: To determine the extent of bone mineral density (BMD) interlimb differences at several hip locations in the involved versus noninvolved lower extremity following anterior cruciate ligament $(\mathrm{ACL})$ surgery.

Background: Disuse following $\mathrm{ACL}$ reconstruction can be extensive. This disuse not only affects the soft tissue, but may also affect the skeletal structure. The extent of this disuse specific to the proximal femur has not been previously determined.

Methods and Measures: BMD was assessed in 15 subjects, 17 to 51 years old, who were between 6 and 32 months post-ACL reconstruction surgery. Bone mineral content (BMC) and BMD of the femoral neck, trochanteric region, intertrochanteric region, and entire hip were measured as a primary emphasis of this study. BMD and BMC of the entire lower extremities were also measured bilaterally.

Results: BMD was significantly less in the involved lower extremity compared to noninvolved lower extremity at several hip sites: $6.6 \%$ less $(P<.001)$ for the trochanteric region, $4.0 \%$ less $(P<.001)$ for the entire hip, and $3.4 \%$ less $(P=.004)$ for the intertrochanteric region. No significant differences were noted comparing the entire lower extremities for either BMD $(0.9 \%$, $P=.48)$ or $\mathrm{BMC}(3.7 \%, P=.09)$.

Conclusion: BMD differences at the hip are significant in patient's postoperative ACL reconstruction, especially in the trochanteric region. J Orthop Sports Phys Ther 2006;36(11):837-844. doi:10.2519/jospt.2006.2278

Key Words: ACL, anterior cruciate ligament, dual energy X-ray absorptiometry, hip

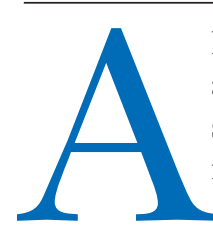

lterations in normal knee function following anterior cruciate ligament (ACL) injury and surgery have been extensively researched. An area that has not been well researched is the extent to which bone mineral density (BMD) changes following ACL reconstruction surgery. ${ }^{1,9,14,20,27,30}$ Previous studies have looked specifically at the knee $\mathrm{k}^{1,14,20,27,30}$ and, to a lesser extent, the spine ${ }^{14,20}$ and the calcaneus. ${ }^{9,16}$ To date, relatively few studies have looked specifically at BMD changes of the hip in these patients, ${ }^{14,15,27}$ and none have looked at the hip as the specific location of emphasis.

\footnotetext{
Assistant Professor, Wichita State University Department of Physical Therapy, Wichita, KS.

2 Staff Physical Therapist, Via Christi Sports and Orthopedic Physical Therapy, Wichita, KS.

${ }^{3}$ Associate Professor, Wichita State University Department of Kinesiology and Sport Studies, Wichita, KS. The protocol of this study was approved by Wichita State University Institutional Review Board, and Via Christi Regional Medical Center Institutional Review Board.

Address correspondence to Michael P. Reiman, Department of Physical Therapy, Wichita State University, 1845 N Fairmount, Wichita, KS 67260-0043.E-mail: michael.reiman@wichita.edu
}

Schmitz $^{27}$ examined multiple sites, including the hip and knee, comparing BMD loss after ACL injury and reconstruction. Two groups with ACL injuries were examined: one group that had not undergone surgical reconstruction and the other that had surgical reconstruction. Both the surgicalreconstruction and conservatively treated groups demonstrated significant losses in BMD at all locations throughout the hip and knee, except the anterior-posterior and lateral projections of the proximal tibia at a mean of 2.5 years postinjury. Other studies have noted considerable and statistically significant loss of bone mass to the affected lower extremity in comparison to conservative treatment for ACL tears at the distal femur, patella, and proximal tibia $^{20}$ as well as at the calcaneus. $^{9,15}$ Anderson and Nilsson $^{1}$ used gamma absorptiometry to measure bone mineral content (BMC) in individuals with clinical signs of ligamentous injuries to the knee joint without any signs of fracture. The authors found lower BMC $(P<.01)$ with both surgical reconstruction and conservative treatment. Again, these measures were localized to the knee.

Kannus et $\mathrm{al}^{14}$ and Karlsson et $\mathrm{al}^{15}$ are the only authors that have assessed hip BMC and BMD. Kan- 
TABLE 1. Characteristics of all participants.



Abbreviation: BPTB, bone-patellar tendon-bone autograft.

*Calculated for 14 subjects included in the study (excluding subject 15).

nus et $\mathrm{al}^{14}$ assessed BMD at several sites from the lumbar spine to the calcaneus. Participants sustaining a severe injury demonstrated an average deficit of $6 \%$ in the distal aspect of the tibia of the involved lower extremity. The only common site in both studies was the femoral neck. Neither study demonstrated significant interlimb differences. Karlsson et $\mathrm{al}^{15}$ did find a significant interlimb difference at the trochanters only. However, no studies have specifically looked at various locations within the hip.

Declines in bone mineral status have been shown to have long-lasting effects. A unique case study by Sievanen et $\mathrm{al}^{30}$ determined an immediate significant decrease (20\%) and an approximate $10 \%$ deficit in specific anatomical location densities when measured using dual-energy X-ray absorptiometry (DEXA) 1 -year postinjury. The DEXA method is a common method of in vivo bone assessment for a variety of reasons, including accuracy, ${ }^{6,31}$ precision, ${ }^{6,31}$ stability, ${ }^{31}$ cost,$^{31}$ subject radiation dose and compliance, ${ }^{6,31}$ freedom to select skeletal sites, ${ }^{6,31}$ as well as speed and ease of scanning for the assessment of bone mineral status. ${ }^{6,31}$

The long-term adaptations to injury should be of concern as the population of subjects with post-ACL reconstruction ages. It is important to understand the future implications of BMD deficit in these subjects. Because osteoporosis is prevalent in areas of high trabecular bone content, ${ }^{7,12}$ and the fact that the hip is a common fracture site in older individuals with osteoporosis, ${ }^{7,12}$ attention should be given to investigating BMD of specific hip locations. DEXA scanning of the femoral neck is one of the best BMD methods to screen for osteoporosis. ${ }^{18}$ The fact that relatively few studies have looked at these specific hip locations ${ }^{14,15,27}$ and the lack of agreement among these studies accentuates the need for further investigation.

Through critical review, no consistent conclusions can be made regarding BMD differences of the hip for the involved and noninvolved lower extremity following ACL reconstruction due to the lack of previous studies. Bone mineral status has not been conclusively determined in the patient post-ACL reconstruction. Therefore, the purpose of this study was to determine the interlimb differences of bone mineral properties, specifically in the hip region, following reconstructive surgery.

\section{METHODS}

\section{Participants}

Participants selected for this study included 15 subjects that had undergone ACL reconstruction within a timeframe of 6 to 32 months prior to the study (Table 1). These participants were recruited from a list of patients having undergone ACL reconstruction and expressed availability to participate in the study. Each of the participants experienced similar surgical procedures by 1 of 3 local surgeons. Participants were excluded if they reported any prior history of bone disease or previous trauma to either hip, as per a signed medical history form. Means, standard deviations, and ranges of subjects' age, mass, and height, as well as participant's sex, involved extremity, lower extremity dominance, time frame from surgery, and graft source are given in Table 1. Table 2 provides descriptive statistics of the individual 
TABLE 2. Descriptive statistics (mean \pm SD, range) of variables analyzed.

\begin{tabular}{lcc}
\hline & Involved Lower Extremity & Noninvolved Lower Extremity \\
\hline BMD lower extremity & $1.42 \pm .21(1.18-1.97)$ & $1.43 \pm .19(1.19-1.92)$ \\
Trochanter BMD & $0.80 \pm .13(.61-1.08)$ & $0.85 \pm .12(.63-1.12)$ \\
Hip BMD & $1.04 \pm .14(.87-1.37)$ & $1.09 \pm .13(.94-1.43)$ \\
Femoral neck BMD & $1.03 \pm .14(.84-1.35)$ & $1.06 \pm .15(.90-1.36)$ \\
Hip intertrochanteric BMD & $1.19 \pm .15(1.02-1.57)$ & $1.23 \pm .16(1.09-1.68)$ \\
BMC lower extremity & $552.19 \pm 115.06(368.60-776.20)$ & $573.11 \pm 110.84(403.50-793.50)$ \\
Hip trochanter BMC & $9.88 \pm 2.31(6.03-14.89)$ & $10.70 \pm 2.79(5.90-16.39)$ \\
Hip BMC & $40.24 \pm 9.22(24.69-52.93)$ & $42.19 \pm 8.49(31.06-57.15)$ \\
Femoral neck BMC & $5.22 \pm 1.02(3.55-7.29)$ & $5.46 \pm 1.06(3.72-7.15)$ \\
Hip intertrochanteric BMC & $25.14 \pm 6.50(15.12-33.75)$ & $26.02 \pm 5.17(19.49-34.39)$ \\
\hline
\end{tabular}

Abbreviations: $\mathrm{BMC}$, bone mineral content $(\mathrm{g})$; $\mathrm{BMD}$, bone mineral density $\left(\mathrm{g} / \mathrm{cm}^{2}\right)$.

variables. Prior to participation, each participant received an explanation of all risks, benefits, and procedures and signed a consent form approved by Wichita State University and Via Christi Regional Medical Center Institutional Review Boards.

\section{Instrumentation and Procedures}

The height and body mass of each participant were measured. The BMC and BMD were measured per standard protocol using a Hologic QDR 4500 Elite series DEXA Bone Densiometer (Bedford, MA). DEXA has been shown to be a very reliable $(r=0.99)$ and precise (coefficient of variation less than 1\%) method for assessing body composition, as well as total body, spine, and femur BMD. ${ }^{23,24}$ The Hologic QDR series has also been shown to demonstrate in vitro accuracy of greater than $96 \%$ at the hip for the 1000 series in a multicenter study. ${ }^{17}$ Specific hip location reliabilities are within $1.3 \%$ at the femoral neck and $0.6 \%$ for the trochanter, ${ }^{21} 2.2 \%$ at the neck and $1.1 \%$ at the trochanter, ${ }^{5}$ and $1.16 \%$ for the trochanter and $1.66 \%$ for the femoral neck. ${ }^{37}$ Quality control (QC) calibrations were conducted before each scan using a Hologic spine phantom, and values were verified to be within \pm 1 standard deviation from the reference mean as determined by Hologic for the unit.

Participants were positioned in the supine position on the scanning table where they received instructions to remain motionless during the procedure (Figure 1). The entire body was placed in the scan field as determined by the outline of the table. The entire body scan required the subject to be lined up symmetrically in the measurement field while in the supine position. The length and width of the entire body scan were approximately 193 and $69 \mathrm{~cm}$, respectively. The length and width of the scan for each hip was approximately 15 and $11 \mathrm{~cm}$, respectively. Separate scans of the entire body and bilateral hips were performed. The time to complete each hip scan was approximately 1 minute and for the entire body scan approximately 7 minutes. All testing, completed by the principal investigator, depended on each partici- pant's time and travel availability to undergo testing. Each participant was tested only once.

The hips were scanned individually. Each hip was lined-up according to protocol and was held in a slightly internally rotated position with a triangularshaped plastic apparatus, according to the manufacturer's instruction manual recommendations. The plastic apparatus strapped to each lower extremity allowed the hip to be optimally positioned for accurate assessment.

BMD (calculated in grams per centimeter squared) and BMC (calculated in grams) were determined using the manufacturer's software. The lower extremities were sectioned for analysis via the manufacturer's recommended protocol. The specific anatomical regions of the hip that were measured were femoral neck, trochanteric region, intertrochanteric region, and entire hip (Figure 2). Measurements and analyses were performed by the principal investigator, who was not blinded to extremity involvement. Training in the specifics of manufacturer's recommended protocol was implemented prior to initiation of the study. This investigator also had 13 years of clinical orthopedic

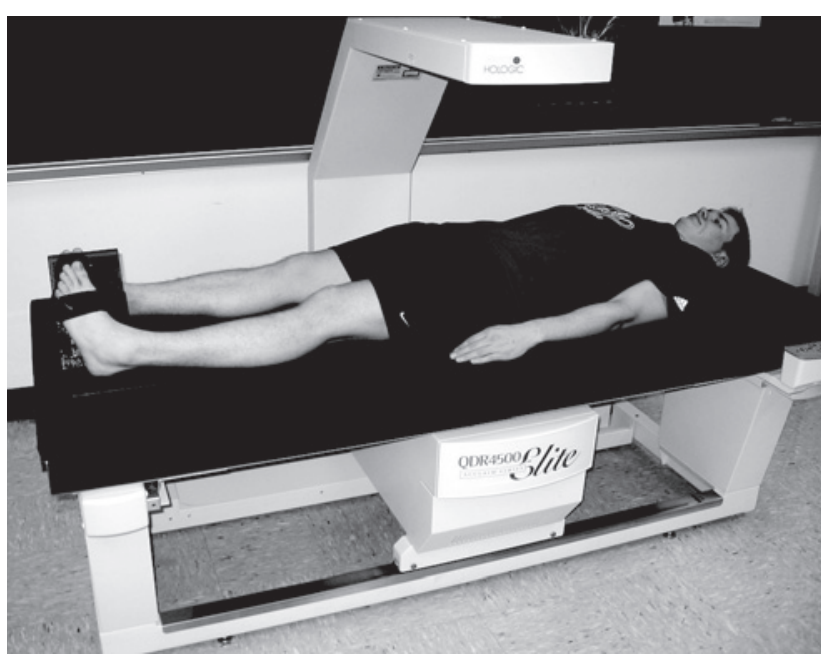

FIGURE 1. Hip scan positioning for dual-energy X-ray absorptiometry (DEXA) analysis (using plastic apparatus for proper hip positioning). 


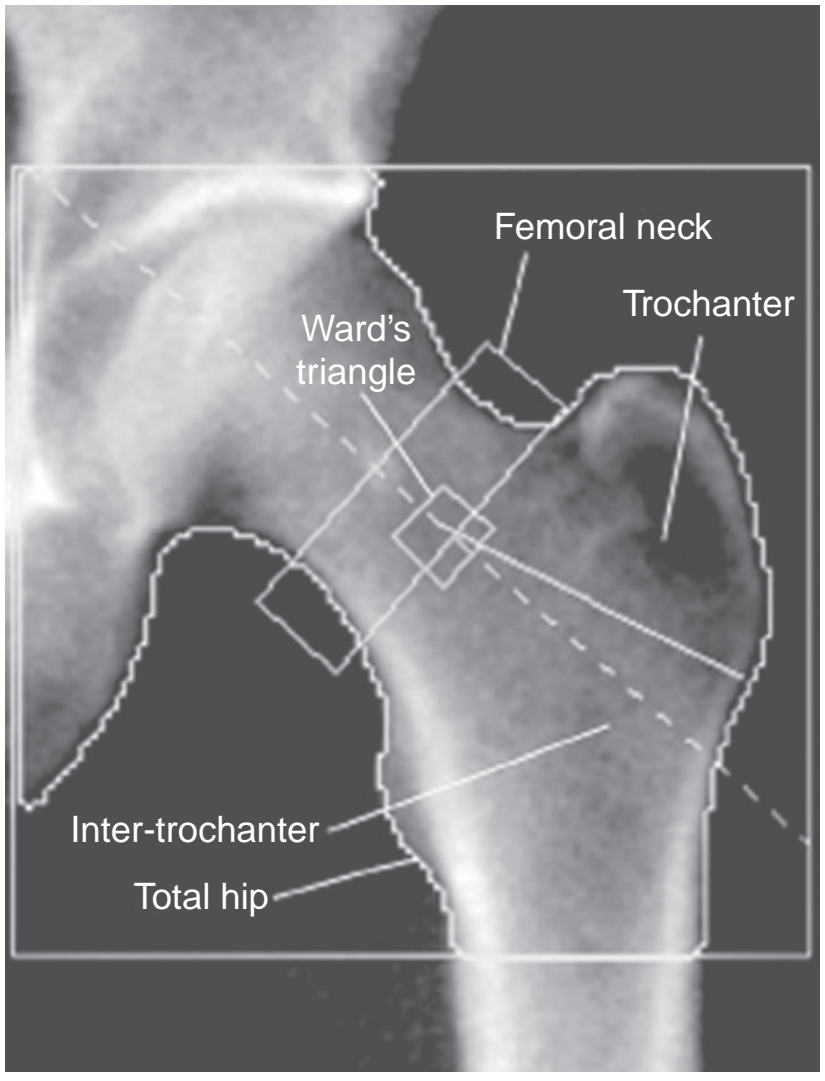

FIGURE 2. Specific locations included in hip analysis. Reprinted with permission from Hologic Inc (Bedford, MA).

physical therapy experience with knowledge of anatomical landmarks used for the protocol.

\section{Data Analysis}

Means and standard deviations were determined for all dependent variables. Paired sample $t$ tests were used to determine differences of the dependent variables between the involved and noninvolved lower extremities. The alpha level was set a priori at .05 for all statistical analyses. However, a Bonferroni adjustment was utilized as a correction for the use of multiple $t$ tests. This correction factor $(0.05 / 5)$ reduced the alpha level to .01. The statistical software package SPSS Version 11.0 (SPSS, Chicago, IL) was used for all analyses.

\section{RESULTS}

Based on the scan results, 1 participant was identified as osteopenic, and all data for that subject were removed prior to analysis (Appendix). Data are presented on the remaining 14 participants (17 to 42 years of age) who completed the study. Means, standard deviations, and ranges of the various BMD and BMC measurements for both the entire lower extremity and at the various hip sites are shown in Table 2.

Statistical analysis of differences between the involved versus noninvolved lower extremities demon- strated that the involved hip BMD was lower than the noninvolved hip BMD at the trochanteric $(6.6 \%$ difference, $P<.001)$ and intertrochanteric $(3.4 \%$ difference, $P=.004$ ) locations, as well as for the entire hip $(4.0 \%$ difference, $P<.001)$ (Table 3$)$. No significant differences in BMC were found for any locations or entire lower extremity measures (BMD or BMC).

\section{DISCUSSION}

The hip is an area of high trabecular bone content and is highly susceptible to osteoporosis and fractures. ${ }^{7,12}$ Sparse information exists regarding BMD and BMC, specifically at the hip following ACL reconstruction surgery. The purpose of this study was to determine, via DEXA, the differences in BMD and $\mathrm{BMC}$ in the involved versus noninvolved lower extremities following ACL reconstruction. Our principal interest was the specific anatomical locations in the hip area due to the lack of consistent findings in this area and, more importantly, the overall lack of investigation of these sites. The primary finding of this study was that hip BMD was lower in the involved versus noninvolved lower extremities in participants 6 to 32 months following ACL reconstruction. No differences existed when comparing entire lower extremity BMD and BMC measurements, as well as with all BMC measurements in the hip region. It should be noted that several of the site-specific comparisons, as well as the entire hip comparison, reached a $P$ level below .05 but failed to reach the Bonferroni-adjusted level of .01.

The differences observed at the various hip sites are in agreement with Schmitz, ${ }^{27}$ who also found a significant difference in the trochanteric region of the involved hip following ACL reconstruction. Significant deficits were reported in BMD of $4.6 \%$ at the trochanteric region of the involved hip versus the noninvolved hip and a $1.1 \%$ difference at the trochanteric region in a group of subjects that were ACL-deficient without having undergone ACL reconstruction. Data from 2 groups of individuals were examined; 1 group had not undergone surgical reconstruction and the other group had undergone surgical reconstruction. The first group consisted of 11 participants who had suffered unilateral ACL injury an average of 8.5 years prior to the time of the study. The ACL reconstruction group included 24 participants having undergone unilateral ACL reconstruction approximately 2.5 years prior to the study. DEXA was used to assess BMD at 3 sites on the hip, 3 sites on the distal femur, 4 sites on the tibia, and a lateral projection of the patella. In both groups, the involved lower extremity was compared to the noninvolved lower extremity. Significant lower BMD at all locations was noted in both groups, except at the anterior-posterior and lateral projections of the proximal tibia. Interestingly, more BMD was lost from the trochanteric hip region, medial femoral condyle, 
TABLE 3. Paired samples test differences (comparison of involved versus noninvolved lower extremities).

\begin{tabular}{llcccc}
\hline & Mean & SD & \% def & $\boldsymbol{t}$ & $\boldsymbol{P}$ \\
\hline Bone mineral density $\left(\mathrm{g} / \mathrm{cm}^{2}\right)$ & & & & & \\
$\quad$ Entire lower extremity & -0.013 & 0.068 & 0.9 & -0.73 & .476 \\
$\quad$ Trochanteric & -0.056 & 0.043 & 6.6 & -4.82 & $<.001$ \\
$\quad$ Entire hip & -0.043 & 0.034 & 4.0 & -4.69 & $<.001$ \\
$\quad$ Neck & -0.035 & 0.054 & 3.3 & -2.46 & .028 \\
$\quad$ Intertrochanteric & -0.042 & 0.044 & 3.4 & -3.53 & .004 \\
Bone mineral content (g) & & & & -1.86 & .086 \\
$\quad$ Entire lower extremity & -20.918 & 42.086 & 3.7 & -2.31 & .038 \\
$\quad$ Trochanteric & -0.816 & 1.319 & 7.6 & -2.91 & .012 \\
Entire hip & -1.946 & 2.503 & 4.6 & -2.56 & .024 \\
$\quad$ Neck & -0.246 & 0.360 & 4.5 & -1.33 & .208 \\
Intertrochanteric & -0.879 & 2.480 & 3.4 & & \\
\hline
\end{tabular}

Abbreviations: \% def, percent of deficit of involved to noninvolved.

and lateral femoral condyle in the group undergoing ACL reconstruction, compared to the group with ACL injury alone.

The only other studies of patients post-ACL reconstruction that have specifically examined the hip for BMD and BMC were Kannus et $\mathrm{al}^{14}$ and Karlsson et al. $^{15}$ Kannus et al $^{14}$ studied only the femoral neck and found a nonsignificant $0.4 \%$ reduction in BMD of the involved lower extremity, as compared to the noninvolved lower extremity. Karlsson et $\mathrm{al}^{15}$ measured the femoral neck, Ward's triangle, and the greater trochanter. A significant difference was found only at the trochanters when comparing the 2 extremities for BMD.

Sievanen et $\mathrm{al}^{30}$ had the unique opportunity to assess BMD prior to and after ACL reconstruction. One-year postinjury, the participant's specific anatomical location bone densities were still approximately $10 \%$ below baseline. An additional follow-up performed 2 years after the injury indicated further recovery, although the preinjury values had not been reached. ${ }^{29}$

Kannus et $\mathrm{al}^{14}$ also noticed a positive correlation between the rating scores to assess function of the knee and BMD deficits at the distal aspect of the femur, patella, and the proximal aspect of the tibia. The authors concluded that the greater the BMD of the involved knee, the better the functional scores of the injured knee on these scales. ${ }^{22,35}$

In comparison, the present study considered multiple locations within the hip (4 each for BMD and BMC), compared to 3 fields in the Schmitz ${ }^{27}$ study and only 1 field in the Kannus et $\mathrm{al}^{14}$ study. The multiple projections were assessed to obtain a more accurate assessment of the hip bone mineral status. Furthermore, the present study, unlike that of Karlsson et $\mathrm{al}^{15}$ compared BMC measurements at all of the hip sites as well as for the entire lower extremity. Although significant differences in specific anatomical locations in and around the knee have been widely reported (and in this study demonstrated at the hip), no significant differences were found for entire lower extremity BMD and BMC measurements.

Results point to differences in the hip comparisons with respect to lower extremity involvement. The cross-sectional design of the study limits conclusions regarding changes that occur in bone status following surgery. It could be speculated that there already was a difference in bone status between the 2 extremities prior to injury due to lower extremity dominance. However, it has previously been shown that there is no difference in BMD between dominant and nondominant lower extremities of uninjured people. ${ }^{13}$ Furthermore, these results may understate the actual loss of bone in the involved side because others have found that BMD was also reduced in the noninvolved lower extremity following surgery, possibly as a result of decreased weight-bearing activity. ${ }^{30,33}$ It should be noted that our study involved a mixture of dominant and nondominant lower-extremity injured limbs.

Subjects selected in the current study had all been rehabilitated with similar protocols by therapists who had similar backgrounds and rehabilitation experience. Rehabilitation should take into account the fact that significant BMD changes were discovered with several different hip-site measurements. Calbet et $\mathrm{al}^{4}$ found a significant increase in BMC in the dominant upper extremity of professional tennis players. While this demonstrates activity-specific increases in BMC, it is interesting to note that these changes were evidenced in an extremity that is predominantly nonweight bearing. In addition, it has been demonstrated that external loading beyond that provided by normal weight-bearing promotes BMC accretion. ${ }^{10}$ There is a positive influence of exercise on bone status as measured by ultrasound and DEXA, specifically at the femoral neck. ${ }^{19}$ BMD is specifically influenced by the type of mechanical loading. ${ }^{3}$ High skeletal impact loading training has been suggested to optimize bone health, specifically at the pelvis and proximal femur., ${ }^{3,10,19,33,34}$ Walking alone had a significant positive effect on BMD for the lumbar spine, but not on 
the femur or calcaneus. ${ }^{25}$ The higher impact level of activity may be necessary for promotion of bone mineral accretion and minimizing bone mineral losses following ACL reconstruction.

Although it has not been specifically determined in the client post-ACL reconstruction, it has been shown in patients with hemiplegia that bone mineral loss is associated with length of immobilization. ${ }^{8}$ Therefore, rehabilitation consisting of strengthening exercises performed in a predominantly weight-bearing manner may be of benefit to bone mineral status. Because weight bearing is considered a major stimulus for bone formation, ${ }^{12,26}$ a lack of normal weight bearing on the involved lower extremity may contribute to decreased bone mineral status of the involved hip as shown in this study. Rehabilitation specialists should take this into consideration when planning the rehabilitation process. Modification of weight bearing might prevent any deleterious effects on the bone mineral status of the involved lower extremity.

Limitations of this study could include the fact that measurements were limited to the hip. However, it might also be argued that the use of hip measurements could be an important parameter. The association between the risk of fracture and low bone mass is well established. ${ }^{7,28,32}$ Due to the hip being an area of high trabecular bone content, it would stand to reason that this risk of osteoporosis and osteoporotic fractures would be much more prevalent in the hip area compared to the knee in these individuals. DEXA measurements, specifically at the femoral neck, are one of the best and most cost-effective BMD methods to screen for osteoporosis. ${ }^{18,24}$ Therefore, it could be argued that hip bone mineral status should be closely monitored, not only in persons post-ACL reconstruction but anyone sustaining any significant damage to the lower appendicular skeleton. Hip BMD may be relevant for the aging patient post-ACL reconstruction as well. It is also known that an increase in the rate of mineral turnover is one of the early responses of bone to trauma as several investigators have reported substantial reductions in bone mass after fracture of long bones, not only at the site of the fracture but also at adjacent sites proximal and distal to it. $^{2}$ Detecting bone turnover in the hip may be an early indicator of future changes to come at the knee following ACL reconstruction.

The fact that measurements were taken in subjects of a wide range could be a limitation of this study. It was not expected that any age-specific changes would affect the results. The fact that side-to-side comparisons were made in all test subjects eliminates any age-related changes.

Also, the timeframe from surgery to measurement was variable (6 to 32 months). In a single-subject case study, Sievanen et $\mathrm{al}^{30}$ reported that timeframe is a significant consideration. This is in direct contradiction to evidence from previously mentioned stud- ies $^{9,14,16}$ which note that time differences between surgery and measurement have little effect, thereby providing an opportunity to increase sample size for this study and improve the generalizability of results. This was not expected to have a substantial influence on the results due to previously mentioned crosssectional and longitudinal studies looking at much longer timeframes than used in the present study. ${ }^{9,14,16}$ Additionally, it has been shown that length of time from injury to surgery did not correlate with bone density loss. ${ }^{16}$

\section{CONCLUSION}

BMD differences in the hip region are significant in patients who are postoperative ACL reconstruction. Measurements at specific hip sites demonstrated greater differences in bone mineral status than measurements of the entire lower extremity. The use of weight-bearing activities to improve bone status for patients post-ACL reconstruction is an area of study that should undergo further testing.

\section{REFERENCES}

1. Andersson SM, Nilsson BE. Changes in bone mineral content following ligamentous knee injuries. Med $\mathrm{SCi}$ Sports. 1979;11:351-353.

2. Andersson SM, Nilsson BE. Changes in bone mineral content following tibia shaft fractures. Clin Orthop Relat Res. 1979;226-229.

3. Bemben DA, Buchanan TD, Bemben MG, Knehans AW. Influence of type of mechanical loading, menstrual status, and training season on bone density in young women athletes. J Strength Cond Res. 2004;18:220-226.

4. Calbet JA, Moysi JS, Dorado C, Rodriguez LP. Bone mineral content and density in professional tennis players. Calcif Tissue Int. 1998;62:491-496.

5. Chilibeck P, Calder A, Sale DG, Webber C. Reproducibility of dual-energy x-ray absorptiometry. Can Assoc Radiol J. 1994;45:297-302.

6. Compston JE. Bone density: BMC, BMD, or corrected BMD? Bone. 1995;16:5-7.

7. Deal CL. Osteoporosis: prevention, diagnosis, and management. Am J Med. 1997;102:35S-39S.

8. Demirbag D, Ozdemir F, Kokino S, Berkarda S. The relationship between bone mineral density and immobilization duration in hemiplegic limbs. Ann Nucl Med. 2005; 19:695-700

9. Ejerhed L, Kartus J, Nilsen R, Nilsson U, Kullenberg R, Karlsson J. The effect of anterior cruciate ligament surgery on bone mineral in the calcaneus: a prospective study with a 2-year follow-up evaluation. Arthroscopy. 2004;20:352-359.

10. Fehling PC, Alekel L, Clasey J, Rector A, Stillman RJ. A comparison of bone mineral densities among female athletes in impact loading and active loading sports. Bone. 1995;17:205-210.

11. Granhed $H$, Jonson $R$, Hansson T. The loads on the lumbar spine during extreme weight lifting. Spine. 1987;12:146-149.

12. Johnston CC, Slemenda C. Osteoporosis: an overview. Phys Sports Med. 1987;15:65-68. 
13. Kannus $P$, Jarvinen $M$, Sievanen $H$, Jarvinen TA, Oja $P$ Vuori I. Reduced bone mineral density in men with a previous femur fracture. J Bone Miner Res. 1994;9:1729-1736.

14. Kannus $P$, Sievanen $H$, Jarvinen $M$, Heinonen $A$, Oja $P$, Vuori I. A cruciate ligament injury produces considerable, permanent osteoporosis in the affected knee. J Bone Miner Res. 1992;7:1429-1434.

15. Karlsson MK, Nilsson BE, Obrant KJ. Bone mineral loss after lower extremity trauma. 62 cases followed for 15-38 years. Acta Orthop Scand. 1993;64:362-364.

16. Kartus J, Stener S, Nilsen R, Nilsson U, Eriksson BI Karlsson J. Bone mineral assessments in the calcaneus after anterior cruciate ligament injury. An investigation of 92 male patients before and two years after reconstruction or revision surgery. Scand J Med Sci Sports. 1998;8:449-455.

17. Khan KM, Henzell SL, Broderick C, et al. Instrument performance in bone density testing at five Australian centres. Aust N Z J Med. 1997;27:526-530.

18. Lafferty FW, Rowland DY. Correlations of dual-energy X-ray absorptiometry, quantitative computed tomography, and single photon absorptiometry with spinal and non-spinal fractures. Osteoporos Int 1996;6:407-415

19. Lehtonen-Veromaa M Mottonen T, Nuotio I, Heinonen OJ, Viikari J. Influence of physical activity on ultrasound and dual-energy X-ray absorptiometry bone measurements in peripubertal girls: a cross-sectional study. Calcif Tissue Int. 2000;66:248-254.

20. Leppala J, Kannus P, Natri A, et al. Effect of anterior cruciate ligament injury of the knee on bone minera density of the spine and affected lower extremity: a prospective one-year follow-Up study. Calcif Tissue Int 1999;64:357-363.

21. Lilley J, Walters BG, Heath DA, Drolc Z. In vivo and in vitro precision for bone density measured by dual energy X-ray absorption. Osteoporos Int. 1991;1:141146.

22. Lysholm J, Gillquist J. Evaluation of knee ligament surgery results with special emphasis on use of a scoring scale. Am J Sports Med. 1982;10:150-154.

23. Mazess RB. Bone densitometry of the axial skeleton. Orthop Clin North Am. 1990;21:51-63.

24. Mazess RB, Barden HS. Measurement of bone by dual-photon absorptiometry (DPA) and dual-energy
X-ray absorptiometry (DEXA). Ann Chir Gynaecol. 1988;77:197-203.

25. Palombaro KM. Effects of walking-only interventions on bone mineral density at various skeletal sites: a metaanalysis. J Geriatr Phys Ther. 2005;28:102-107.

26. Pirnay F, Bodeux M, Crielaard JM, Franchimont P. Bone mineral content and physical activity. Int J Sports Med. 1987;8:331-335.

27. Schmitz MA. Research links ACL injury to bone density loss. Biomechanics. 2001;8:93-98.

28. Schoutens A, Laurent E, Poortmans JR. Effects of inactivity and exercise on bone. Sports Med. 1989;7:71-81.

29. Sievanen H, Heinonen A, Kannus P. Adaptation of bone to altered loading environment: a biomechanical approach using X-ray absorptiometric data from the patella of a young woman. Bone. 1996;19:55-59.

30. Sievanen $\mathrm{H}$, Kannus $\mathrm{P}$, Heinonen A, Oja P, Vuori I. Bone mineral density and muscle strength of lower extremities after long-term strength training, subsequent knee ligament injury and rehabilitation: a unique 2-year follow-up of a 26-year-old female student. Bone. 1994;15:85-90.

31. Sievanen H, Oja P, Vuori I. Precision of dual-energy x-ray absorptiometry in determining bone mineral density and content of various skeletal sites. J Nucl Med. 1992;33:1137-1142.

32. Sinaki M. Exercise and osteoporosis. Arch Phys Med Rehabil. 1989;70:220-229.

33. Taaffe DR, Marcus R. The muscle strength and bone density relationship in young women: dependence on exercise status. J Sports Med Phys Fitness. 2004;44:98103.

34. Taaffe DR, Robinson TL, Snow CM, Marcus R. Highimpact exercise promotes bone gain in well-trained female athletes. J Bone Miner Res. 1997;12:255-260.

35. Tegner Y, Lysholm J. Rating systems in the evaluation of knee ligament injuries. Clin Orthop Relat Res. 1985;4349.

36. Van der Wiel HE, Lips $P$, Nauta J, Patka P, Haarman HJ, Teule GJ. Loss of bone in the proximal part of the femur following unstable fractures of the leg. J Bone Joint Surg Am. 1994;76:230-236.

37. White J, Harris SS, Dallal GE, Dawson-Hughes B. Precision of single vs bilateral hip bone mineral density scans. J Clin Densitom. 2003;6:159-162. 


\section{Appendix}

Scan of Participant 15 Who Was Excluded From the Study Due to Osteopenia
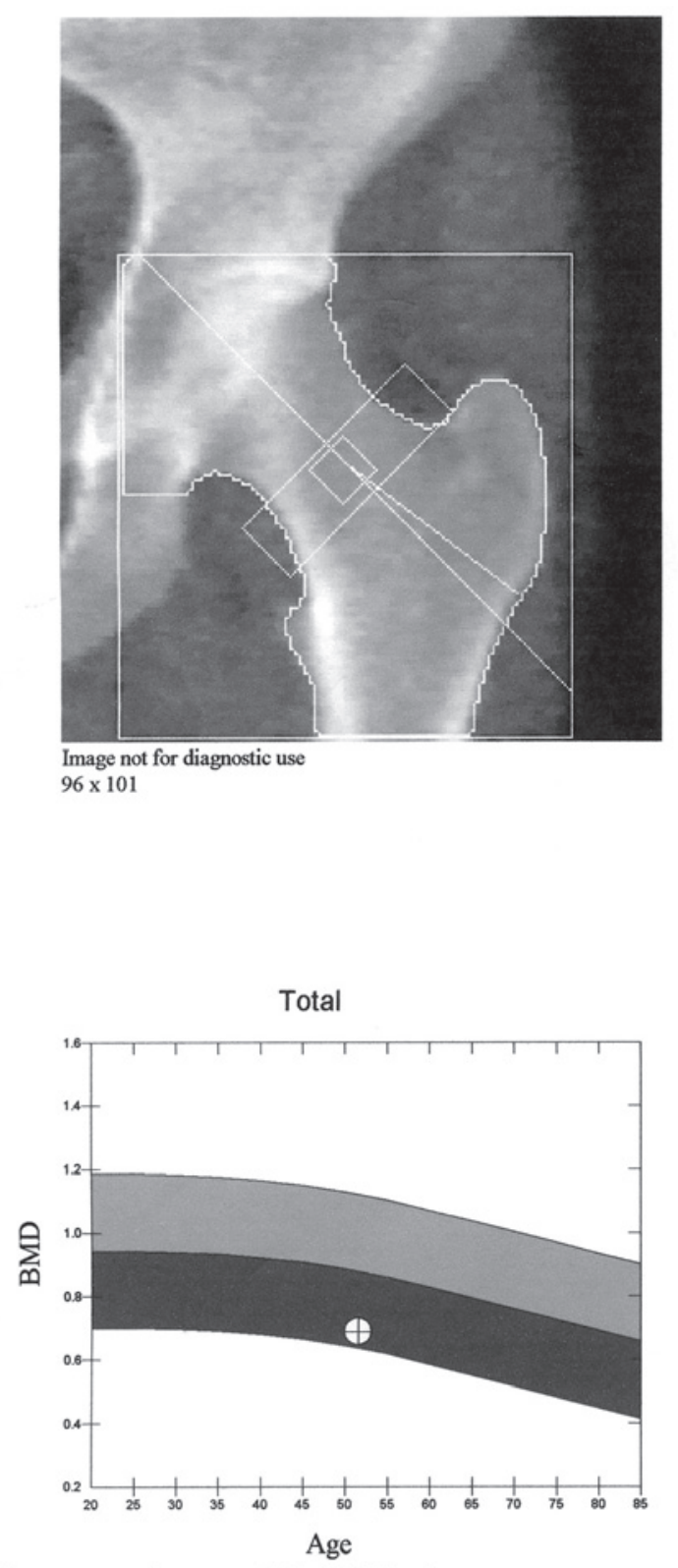

Reference curve and scores matched to White Female

\section{Scan Information:}

Scan Date: March 27, 2003

Scan Type: f Left Hip

Analysis: $\quad$ March 08, 2006 10:44 Version 12.3

Left Hip

Operator: MR

Model: $\quad$ QDR 4500W (S/N 49183)

Comment:

\section{DXA Results Summary:}

\begin{tabular}{|lrrrrr|}
\hline Region & $\begin{array}{r}\text { Area } \\
\left(\mathbf{c m}^{2}\right)\end{array}$ & $\begin{array}{r}\mathbf{B M C} \\
\mathbf{( g )}\end{array}$ & $\begin{array}{r}\mathbf{B M D} \\
\left(\mathbf{g} / \mathbf{c m}^{\mathbf{2}}\right)\end{array}$ & $\begin{array}{r}\mathbf{T}- \\
\text { Score }\end{array}$ & $\begin{array}{r}\mathbf{Z}- \\
\text { Score }\end{array}$ \\
Neck & 5.26 & 3.20 & 0.608 & -2.2 & -1.3 \\
Troch & 10.58 & 5.04 & 0.476 & -2.2 & -1.7 \\
Inter & 15.57 & 13.36 & 0.858 & -1.6 & -1.2 \\
Total & $\mathbf{3 1 . 4 0}$ & $\mathbf{2 1 . 5 9}$ & $\mathbf{0 . 6 8 8}$ & $\mathbf{- 2 . 1}$ & $\mathbf{- 1 . 6}$ \\
Ward's & 1.12 & 0.53 & 0.478 & -2.2 & -0.8 \\
\hline
\end{tabular}

Total BMD CV $1.0^{\circ}$

WHO Classification: Osteopenia

Fracture Risk: Increased

\section{Physician's Comment:}

\section{HOLOGIC}

